\title{
Surface-Enhanced Raman Spectroscopy of Chernozem Humic Acid and Their Fractions Obtained by Coupled Size Exclusion Chromatography-Polyacrylamide Gel Electrophoresis (SEC- PAGE)
}

\author{
S. SANCHEZ-CORTES,* G. CORRADO, O. E. TRUBETSKAYA, O. A. TRUBETSKOJ, \\ B. HERMOSIN, and C. SAIZ-JIMENEZ \\ Instituto de Estructura de la Materia, Consejo Superior de Investigaciones Cientificas, Serrano 121, 28006 Madrid, Spain (S.S.-C., G.C.); Branch \\ of Shemyakin and Ovchinnikov Institute of Bioorganic Chemistry, Russian Academy of Sciences, 142290 Pushchino, Moscow region, Russia \\ (O.E.T.); Institute of Basic Biological Problems, Russian Academy of Sciences, 142290 Pushchino, Moscow region, Russia (O.A.T.); and Instituto \\ de Recursos Naturales y Agrobiologia, Consejo Superior de Investigaciones Cientificas, Apartado 1052, 41080 Sevilla, Spain (B.H., C.S.-J.)
}

\begin{abstract}
A humic acid extracted from a chernozem soil was fractionated combining size exclusion chromatography and polyacrylamide electrophoresis (SECPAGE). Three fractions named $\mathrm{A}, \mathrm{B}$, and $\mathrm{C}+\mathrm{D}$, with different electrophoretic mobilities and molecular sizes (MS), were obtained and subsequently characterized by thermochemolysis and surface-enhanced Raman spectroscopy (SERS). The data confirmed that fraction A, with the higher MS, was more aliphatic than fractions $B$ and $C+D$ and, in turn, fractions with lower MS $(B$ and $C+D)$ denoted an enrichment in lignin residues. These structural features explain conformational changes when varying the $\mathrm{pH}$ in the humic fraction $\mathrm{A}$ and indicated that combination of the two techniques is a good approach for characterizing humic substances.
\end{abstract}

Index Headings: Surface-enhanced Raman spectroscopy; SERS; Humic acid; Chernozem soil; Size exclusion chromatography; Polyacrylamide electrophoresis; SEC-PAGE; Thermochemolysis.

\section{INTRODUCTION}

Natural soil and water humic substances (HS) are the most representative part of stable organic carbon in the biosphere, comprising approximately $60-70 \%$ of the total organic carbon in soils and $60-90 \%$ of dissolved organic carbon in natural waters.

Humic acids (HA) are a subclass of HS. They are the most abundant in the upper $30-60 \mathrm{~cm}$ of the Earth's crust, where they play one of the main roles in regulation of the mobility and fate of plant nutrients and environmental contaminants. Their molecular weights (MW) range from a few hundred to several hundred thousand Daltons and their highly heterogeneous structures and functionalities are the major hindrance for the elucidation of molecular structures of HA. Fractionation and characterization of less complex fractions are the key for a better understanding of their functional role in the environment. Size exclusion chromatography (SEC) and electrophoresis are often used to fractionate HS due to their rapidity, sensitivity, reproducibility, and availability. ${ }^{1-5}$ Recently, Trubetskoj et al. ${ }^{6}$ developed an effective method for the fractionation of soil HA based on the combination of SEC with polyacrylamide gel electrophoresis (PAGE), named SEC-PAGE. Optimal chromatographic and electrophoretic conditions were established to obtain preparative quantities of soil HA fractions, named A, B, and $\mathrm{C}+\mathrm{D}$, with different electrophoretic mobilities (EM) and

Received 4 July 2005; accepted 20 October 2005.

* Author to whom correspondence should be sent. E-mail: imts158@ iem.cfmac.csic.es. molecular sizes (MS). The addition of denaturing agents such as urea at a concentration of $7 \mathrm{M}$ breaks up associations between humic aggregates for both SEC and PAGE. This procedure also eliminates adsorptive hydrogen bonding interactions with the fractionating media. Therefore, the fractionation procedure separates disaggregated primary humic structures. In our preliminary investigation, SEC-PAGE allowed the fractionation of several soil HAs of different genesis and we obtained HA fractions with clear differences in terms of ultraviolet (UV)-visible spectra, fluorescence properties, photochemical activity, and amino acid content. ${ }^{7,8}$

Spectroscopic and structural analyses of HS have experienced a great deal of growth over recent years due to the development of high field magnets and an array of multidimensional techniques. The use of heteronuclear and multidimensional nuclear magnetic resonance (NMR) spectroscopy for mapping the chemical structures and organization of HS provided considerable progress on these complex environmental samples. ${ }^{9}$ Evidence of the primary molecular structure in HS was obtained by Simpson et al., ${ }^{10}$ who stated that the major molecular structural components were aliphatic acids, ethers, esters, and alcohols; aromatic lignin derived fragments; and polysaccharides and polypeptides. The same structural components were evidenced using thermochemolysis, also known as thermally assisted hydrolysis and methylation. ${ }^{11}$

Surface-enhanced Raman spectroscopy (SERS) is a technique that allows the Raman vibrational study of highly fluorescent macromolecules in aqueous solution. ${ }^{12-14}$ This technique made possible the application, for the first time, of the Raman vibrational technique in the characterization of HS, ${ }^{15,16}$ an impossible task by conventional Raman procedures, due to the high fluorescence of these substances. This technique is based on the enormous enhancement of the Raman signal produced by a molecule placed on a rough metallic surface. Moreover, the short-range enhancement predicted by the electromagnetic model of SERS can be used to investigate the electrodynamical behavior of macromolecules adsorbed on charged metal surfaces due to the predominant enhancement of those modes in direct contact with the metal. ${ }^{17}$

In this work we report the analysis of a chernozem HA and its fractions $\mathrm{A}, \mathrm{B}$, and $\mathrm{C}+\mathrm{D}$ obtained by SEC-PAGE using two different and complementary techniques: thermochemolysis and SERS. It is hoped that some structural clues can be obtained from this double analytical approach when applied to 
less complex fractions resulting from the PAGE-SEC fractionation of the bulk HA sample.

\section{MATERIALS AND METHODS}

Soil Sample and Isolation of Humic Acid. The soil sample was taken from the A horizon of a chernozem soil, Kursk region, Russia. Methods for humic acid extraction and purification are reported elsewhere. ${ }^{18}$

Fractionation of Humic Acid. Fractionation of soil HA by SEC-PAGE (i.e., using PAGE for subsequent testing of SEC aliquots from different sections of elution profile) has been previously reported. ${ }^{6}$ Briefly: $10 \mathrm{mg}$ sample of HA was dissolved in $7 \mathrm{M}$ urea and loaded onto a Sephadex G-75 (Pharmacia, Sweden) column $(1.5 \times 100 \mathrm{~cm})$, equilibrated with the same solution. The void $\left(V_{0}\right)$ and the total $\left(V_{\mathrm{t}}\right)$ column volumes were 47 and $160 \mathrm{~mL}$, respectively. The void volume was determined using Dextran Blue 2000. Fractionation ranges for Sephadex G-75 were 80000 to 3000 for proteins and 50000 to 1000 for polysaccharides. Flow rate was $15 \mathrm{~mL} \mathrm{~h}^{-1}$. The UV detector (ISCO, Lincoln, NE) was at $280 \mathrm{~nm}$. Column effluent was collected as $2 \mathrm{~mL}$ aliquots using a collector of fractions (ISCO) and each third aliquot was assayed by PAGE in the presence of denaturating agents according to Trubetskoj et al. ${ }^{19}$ The apparatus was a vertical electrophoresis device (LKB 2001 Vertical Electrophoresis, Sweden) with gel slab $(20 \times 20 \mathrm{~cm})$. Electrophoresis was carried out in $10 \%$ PAG at room temperature for $1 \mathrm{~h}$ at a current intensity of $25 \mathrm{~mA}$. As the gel buffer, $89 \mathrm{mM}$ trisborate, $\mathrm{pH} 8.3$, with $1 \mathrm{mM}$ EDTA and $7 \mathrm{M}$ urea was used. The sample buffer $(0.05 \mathrm{~mL})$ contained $89 \mathrm{mM}$ tris-borate, $\mathrm{pH}$ 8.3, $7 \mathrm{M}$ urea, $1 \%$ SDS, and $1 \mathrm{mM}$ EDTA. On the basis of PAGE analysis, three fractions, $\mathrm{A}, \mathrm{B}$, and $\mathrm{C}+\mathrm{D}$, were obtained from HA samples. Fractions were dialyzed for seven days against distilled water (cut-off of the dialysis membrane was 5000), lyophilized, and used for further physico-chemical analyses. Based on ultrafiltration data, ${ }^{6}$ the molecular weights $3 \times 10^{5}-10^{5}, 10^{5}-3 \times 10^{4}$, and $3 \times 10^{4}-5 \times 10^{3}$ Daltons were found for fractions $\mathrm{A}, \mathrm{B}$, and $\mathrm{C}+\mathrm{D}$, respectively. For obtaining of preparative quantities of fractions, the fractionation procedure was repeated 10 times.

The weight of each fraction was calculated by direct weighing after chromatography. The weight distribution of fractions was calculated using the ratio $W_{\mathrm{i}} / \Sigma W_{\mathrm{i}}$, where $W_{\mathrm{i}}$ is the weight of the fraction and $\Sigma W_{\mathrm{i}}$ is the weight of all fractions obtained after SEC fractionation of HA.

Chemical Analysis. C, H, and N analyses were performed in a Perkin Elmer CHN Analyzer, series II 2400. Values reported were on a moisture- and ash-free basis. The accuracy of the measurements indicated in Table I was $95 \%$ or better.
Thermochemolysis of Humic Acid and its Fractions. Thermochemolysis, or thermally assisted hydrolysis and methylation, was performed in a Fisons gas chromatograph GC 8000/mass detector MD 800 coupled to a Fischer 0316 Curie-point pyrolyzer, using a $30 \mathrm{~m} \times 0.25 \mathrm{~mm}$ SPB-5 column (film thickness $0.25 \mu \mathrm{m}$ ). The $\mathrm{GC}$ oven was kept at $25^{\circ} \mathrm{C}$ by a cryogenic unit and programmed to rise to $2800^{\circ} \mathrm{C}$, at a rate of 5 ${ }^{\circ} \mathrm{C} \min ^{-1}$. The final temperature was held for $20 \mathrm{~min}$. The sample was deposited in a Curie-point small hollow ferromagnetic cylinder (temperature $500{ }^{\circ} \mathrm{C}$ ) and wetted with $5 \mu \mathrm{L}$ of a $25 \%$ by weight aqueous solution of TMAH. The cylinder was slightly dried with $\mathrm{N}_{2}$ and immediately inserted in the pyrolyzer.

The compounds were identified by comparison of the mass spectra with a self-compiled data bank of compounds from a variety of samples. ${ }^{11,20,21}$ In some cases, identification was achieved by computer analysis from a National Bureau of Standards library of about 54000 spectra, with the computer matching being checked against standards whenever possible.

Surface-Enhanced Raman Spectroscopy Analysis of Humic Acid and Its Fractions. The adsorption studies of the humic substances were carried out on a silver colloid prepared by chemical reduction of $\mathrm{Ag}^{+}$with hydroxylamine, following the method described by Leopold and Lendl: ${ }^{22} 4.5$ $\mathrm{mL}$ of a sodium hydroxide solution $(0.1 \mathrm{M})$ were added to 5 $\mathrm{mL}$ of a $6 \times 10^{-2} \mathrm{M}$ hydroxylamine hydrochloride solution. Then, the mixture was added rapidly to a $1.11 \times 10^{-3} \mathrm{M}$ of a silver nitrate aqueous solution, shaking it until a homogeneous mixture was obtained. The resulting colloid showed a milky gray color. The stability of the colloid prepared in this way was lower than the citrate one, due to the formation of nitrogen oxides as a consequence of the reduction process of silver nitrate. Because of the reaction of these oxides with water, the $\mathrm{pH}$ of the Ag-hydroxylamine colloids tends to slightly decrease with the time. Therefore, aqueous $\mathrm{NaOH}$ was added to the final solution in order to bring the $\mathrm{pH}$ to 6.5.

In order to become active, Ag-hydroxylamine colloids were aggregated by addition of potassium nitrate. This aggregation consisted of the nanoparticles approaching to form others with a larger size. This process is necessary in the case of using these systems as substrate for SERS, since specific morphological requirements are needed to enhance the electromagnetic field in the metal surface. This activation is a prerequisite for SERS spectra to be observed at a higher intensity, as we have demonstrated in other works. ${ }^{23,24}$

Samples for Raman measurements were prepared by adding $10 \mu \mathrm{L}$ of an aqueous humic substance solution, prepared by dissolving $1 \mathrm{mg}$ of the corresponding humic fraction in $1 \mathrm{~mL}$ of tri-distilled water to $1 \mathrm{~mL}$ of the silver colloid. The $\mathrm{pH}$ of the

TABLE I. Carbon content (in atomic \%), atomic ratios, and relative amounts in weight of chernozem HA and its fractions obtained by SEC-PAGE (results obtained on an ash- and moisture-free basis).

\begin{tabular}{|c|c|c|c|c|c|c|}
\hline & $\mathrm{C}$ & $\mathrm{H} / \mathrm{C}$ & $\mathrm{C} / \mathrm{N}$ & Ash (\%) & Water $(\%)$ & $\begin{array}{l}\text { Relative amounts } \\
\text { in weight (\%) }\end{array}$ \\
\hline HA & 5.21 & 0.89 & 13.36 & 5.4 & 9.6 & \\
\hline Fraction $\mathrm{C}+\mathrm{D}$ & 4.75 & 0.99 & 15.83 & 9.4 & 10.2 & 36 \\
\hline Remaining fraction ${ }^{\mathrm{a}}$ & $\mathrm{N} \mathrm{d}^{\mathrm{b}}$ & $\mathrm{Nd}$ & $\mathrm{N} \mathrm{d}$ & $\mathrm{N} \mathrm{d}$ & $\mathrm{N} \mathrm{d}$ & 21 \\
\hline
\end{tabular}

${ }^{\text {a }}$ This remaining fraction corresponds to a mixture of fractions $\mathrm{B}$ and $\mathrm{C}+\mathrm{D}$.

${ }^{\mathrm{b}}$ Not determined. 
Humic acid

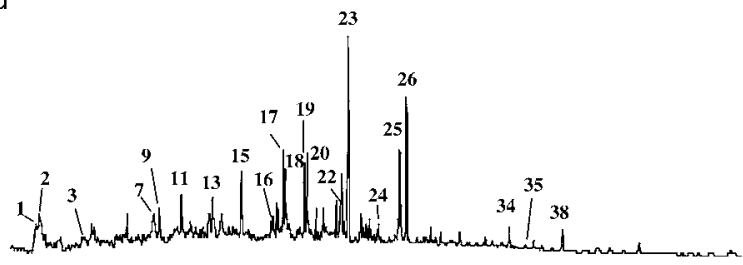

Fraction A

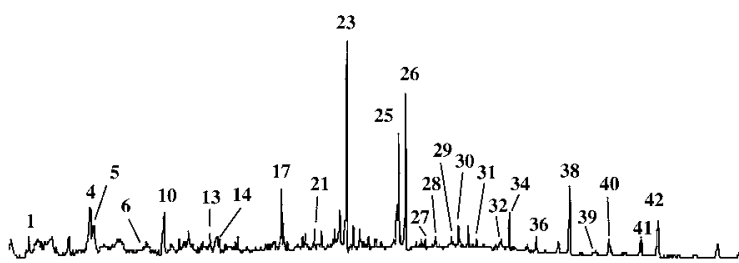

Fraction B

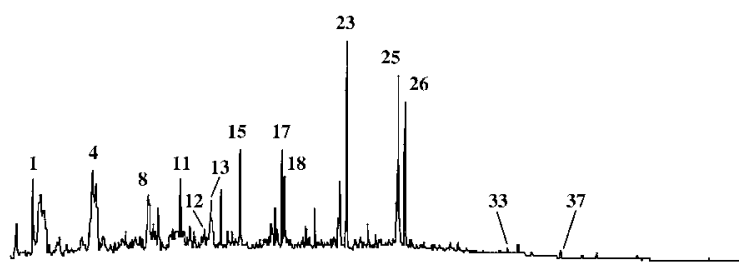

Fraction $\mathrm{C}+\mathrm{D}$

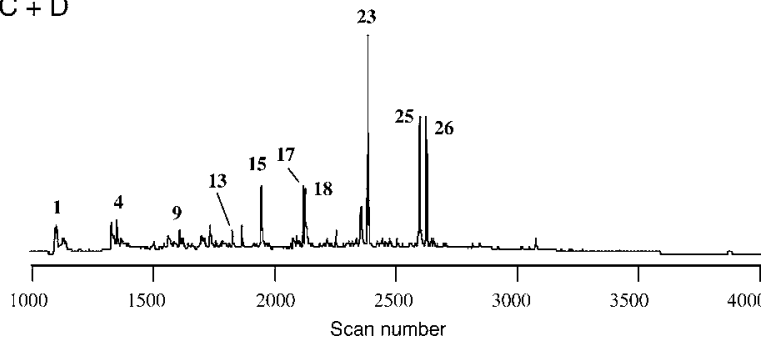

FIG. 1. Chromatograms of chernozem HA and its fractions.

mixture was adjusted by adding $0.1 \mathrm{M} \mathrm{NaOH}$ to $\mathrm{pH} 7$ or 12 . All solutions were prepared with tri-distilled water.

The SERS spectra were recorded with a Renishaw RM2000 Raman spectrometer. As the excitation line we employed the $514.5 \mathrm{~nm}$ line provided by an $\mathrm{Ar}^{+}$laser. The samples were measured in a quartz cell with a $1 \mathrm{~cm}$ optical path length placed in a macro-sampling accessory with a focalization lens of 15 $\mathrm{mm}$. The laser power at the sample was $2 \mathrm{~mW}$. The resolution was set at $2 \mathrm{~cm}^{-1}$ and the geometry of micro-Raman measurements was $180^{\circ}$.

Difference spectra between the SERS recorded at alkaline and neutral $\mathrm{pH}$ was done by taking as a reference the water broad band at $3420 \mathrm{~cm}^{-1}$.

\section{RESULTS}

Thermochemolysis. In this paper the HA and its fractions $\mathrm{A}, \mathrm{B}$, and $\mathrm{C}+\mathrm{D}$ obtained by SEC-PAGE were studied in the scan range 1000-4000 amu, considered the most informative, because this range provides insights on the original components and their chemical structure. ${ }^{25}$

The chromatograms of the thermochemolysates of HA and its fractions $\mathrm{A}, \mathrm{B}$, and $\mathrm{C}+\mathrm{D}$ are displayed in Fig. 1. The major compounds are listed in Table II. Three classes of compounds constituted the vast majority of the identified products: fatty acids, aromatic compounds, and nitrogen derivatives.
TABLE II. Major compounds identified in thermochemolysates of humic acids and fractions.

\begin{tabular}{ll}
\hline & \multicolumn{1}{c}{ Compound } \\
\hline 1 & Benzoic acid, methyl ester \\
2 & Guaiacol \\
3 & Methylguaiacol \\
4 & 1,3 -Dimethyl-2,4 (1H,3H)-Pyrimidinedione \\
5 & $3,4-$ Dimethoxytoluene \\
6 & Vinylguaiacol \\
7 & $3,4-$-Dimethoxystyrene \\
8 & 1,3,5-Trimethyl-2,4(1H,3H)-pyrimidinedione \\
9 & 4-Methoxybenzoic acid, methyl ester \\
10 & 1,3,5-Trimethyl-1,3,5-triazine-2,4,6-(1H,3H,5H)-trione \\
11 & 2-Methyl-1H-isoindole-1,3(2H)-dione \\
12 & $1,2,3$-Trimethoxybenzene \\
13 & Dodecanoic acid, methyl ester $+1,2-$-Benzenedicarboxylic acid, \\
& $\quad$ dimethyl ester \\
14 & 4-Hydroxy-3-methoxybenzoic acid, methyl ester \\
15 & $3,4-$ Dimethoxybenzoic acid, methyl ester \\
16 & 3-(4-Methoxyphenyl)-2-propenoic acid, methyl ester \\
17 & $n$-Tetradecanoic acid, methyl ester \\
18 & 3,4,5-Trimethoxybenzoic acid, methyl ester \\
19 & iso-Pentadecanoic acid, methyl ester \\
20 & anteiso-Pentadecanoic acid, methyl ester \\
21 & $n$-Pentadecanoic acid, methyl ester \\
22 & 3-(3,4-Dimethoxyphenyl)-2-propenoic acid, methyl ester \\
23 & $n$-Hexadecanoic acid, methyl ester \\
24 & $n$-Heptadecanoic acid, methyl ester \\
25 & $n$-Octadecenoic acid, methyl ester \\
26 & $n$-Octadecanoic acid, methyl ester \\
27 & $n$-Docosane \\
28 & $n$-Nonadecanoic acid, methyl ester \\
29 & $n$-Tricosane \\
30 & $n$-Eicosanoic acid, methyl ester \\
31 & $n$-Tetracosane \\
32 & $n$-Heneicosanoic acid, methyl ester \\
33 & $n$-Pentacosane \\
34 & $n$-Docosanoic acid, methyl ester \\
35 & $n$-Hexacosane \\
36 & $n$-Tricosanoic acid, methyl ester \\
37 & $n$-Heptacosane \\
38 & $n$-Tetracosanoic acid, methyl ester \\
39 & $n$-Octacosane \\
40 & $n$-Pentacosanoic acid, methyl ester \\
41 & $n$-Nonacosane \\
42 & $n$-Hexacosanoic acid, methyl ester \\
\hline &
\end{tabular}

The soil HA samples were characterized by the abundance of a series of $n$-fatty acids, from $\mathrm{C}_{12}$ to $\mathrm{C}_{26}$ (Fig. 1 , Table II). The most abundant aliphatic compounds were the $\mathrm{C}_{16}$ and $\mathrm{C}_{18}$ members (peaks 23 and 26, respectively). The unsaturated $\mathrm{C}_{18}$ fatty acid (peak 25) and some branched fatty acids (peaks 19 , 20) were also evidenced. In addition, the $n$-fatty acid $\mathrm{C}_{22}-\mathrm{C}_{26}$ members (peaks $34,36,38,40$, and 42 , respectively) were comparatively abundant in the fraction $\mathrm{A}$ with respect to fractions $\mathrm{B}$ and $\mathrm{C}+\mathrm{D}$, in which they rarely reached importance. A less abundant series of compounds, such as the $n$-alkanes in the range $\mathrm{C}_{22}-\mathrm{C}_{29}$, and aliphatic dicarboxylic acids (not shown in the traces) were minor compounds.

Fatty acid distribution was not homogeneous among HA fractions. In fact, fraction A contained considerably more $n$ fatty acids $>\mathrm{C}_{22}$, derived from plant waxes, than fractions $\mathrm{B}$ and $\mathrm{C}+\mathrm{D}$, where they were found as trace compounds. Although ubiquitous lipids ( $n$-fatty acids $<\mathrm{C}_{22}$ ) were distributed in all fractions, the largest relative amounts appeared primarily associated with fraction A. 
Among the aromatic compounds, phenolic and benzenecarboxylic acids were identified. Lignin thermochemolysis products were represented by benzoic acid methyl ester (peak 1), guaiacol (peak 2), methylguaiacol (peak 3), 3,4-dimethoxytoluene (peak 5), vinylguaiacol (peak 6), 3,4-dimethoxystyrene (peak 7), 4-methoxybenzoic acid methyl ester (peak 9), 1,2,3trimethoxybenzene (peak 12), 4-hydroxy-3-methoxybenzoic acid (vanillic acid) methyl ester (peak 14), 3,4-dimethoxybenzoic acid (veratric acid) methyl ester (peak 15), 3-(4methoxyphenyl)-2-propenoic acid methyl ester (peak 16), 3,4,5-trimethoxybenzoic acid methyl ester (peak 18), and 3(3,4-dimethoxyphenyl)-2-propenoic acid methyl ester (peak 22). The presence of benzoic acid derivatives indicates a lignin biodegradation process through oxidation of the $\mathrm{C}_{3}$-alkyl chain. $^{26}$

Benzene(poly)carboxylic acids included 1,2-benzenedicarboxylic acid (peak 13) as a major compound (Table II), although some other minor compounds (isomers of benzenetriand tetracarboxylic acids) were identified (not shown in the traces). Benzene(poly)carboxylic acids constituted a significant part of the thermochemolysis products in soil fulvic and humic acids. ${ }^{11,20}$

The number of lignin thermochemolysis products encountered indicates that the largest proportion of lignin products was associated with fraction $\mathrm{B}$, denoting a lignin enrichment in this fraction, in agreement with the findings of Saiz-Jimenez et al. ${ }^{18}$ Although lignin products were present in all fractions, the smallest proportion was generally associated with fraction A.

Some nitrogen-containing compounds such as dimethyl- and trimethylpyrimidinediones (peaks 4 and 8 , respectively), trimethyltriazinetrione (peak 10), and methylisoindoledione (peak 11) were identified (Table II). However, the origin of these nitrogen derivatives in our samples is doubtful. The increased amount of $\mathrm{N}$-compounds in all the fractions with respect to the unfractionated HA suggests that the origin of these compounds could be attributed to urea residues immobilized during the fractionation procedure and further reaction involving cyclization in the thermal process.

Surface-Enhanced Raman Spectra. Surface-enhanced Raman spectra of the high MS fraction A at neutral and alkaline $\mathrm{pH}$ are shown in Fig. 2. The SERS spectrum at alkaline $\mathrm{pH}$ (Fig. 2a) is much more intense than the spectrum recorded at neutral conditions (Fig. 2b) and characterized by the presence of two intense and narrow bands appearing at 1308 and $1610 \mathrm{~cm}^{-1}$. These bands can be assigned to ring stretching motions of aromatic compounds, ${ }^{27}$ and they can also be observed in the SERS spectra of hydroxybenzoic acids and in polycyclic aromatic hydrocarbon compounds. Other less intense bands appeared at 1580 and $1360 \mathrm{~cm}^{-1}$, and can be attributed to ring stretching and symmetric $v\left(\mathrm{COO}^{-}\right)$vibrations, respectively, at $1267 \mathrm{~cm}^{-1}$, corresponding to $\mathrm{v}(\mathrm{C}-\mathrm{O})$ motion in phenols, and at $1171 \mathrm{~cm}^{-1}$, assigned to bending vibrations of aromatic $\mathrm{C}-\mathrm{H}$ groups.

At pH 7 (Fig. 2b) two very broad bands were centered at about 1590 and $1380 \mathrm{~cm}^{-1}$. These features can be due to carbon bands caused by the partial thermal degradation of the humic materials. The differences found between the SERS spectra obtained at different $\mathrm{pH}$ values can be observed in the difference spectrum (Fig. 2c), indicating the marked increase of bands at 1610,1308 , and $1248 \mathrm{~cm}^{-1}$ at alkaline $\mathrm{pH}$.

In contrast, the intermediate MS fraction B shows a lower difference in the intensity of the bands appearing at alkaline $\mathrm{pH}$

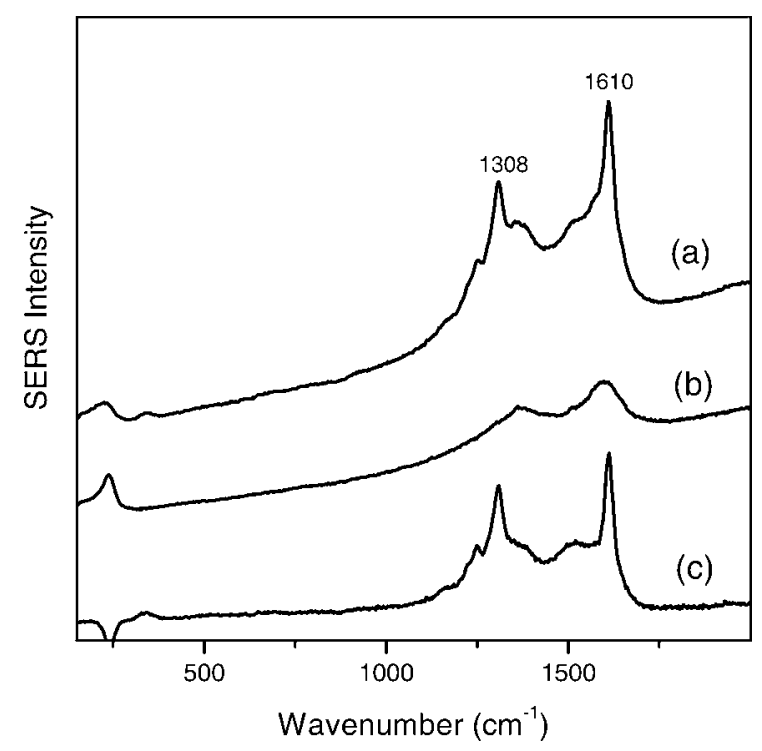

FIG. 2. SERS spectra of the A fraction at (a) alkaline and (b) neutral pH; (c) the difference between (a) and (b).

(Fig. 3a), and those observed at neutral pH (Fig. 3b), which could be related to the higher amount of lignin products in this fraction as revealed by electrophoresis and thermochemolysis data. $^{18,25}$ The molecular matrices forming part of this fraction are more open at neutral $\mathrm{pH}$, as deduced from their different composition. The difference spectrum (Fig. 3c) shows positive intense bands at 1517,1380 , and $1174 \mathrm{~cm}^{-1}$, while the band at $1308 \mathrm{~cm}^{-1}$ is more intense than that at $1610 \mathrm{~cm}^{-1}$, in agreement with a different composition, in particular, a larger amount of oxygenated groups (carboxylate, phenols, ethers, and aromatic moieties in general with a large number of substituents) are deduced from the difference spectrum. The spectrum is similar to the SERS of humic acids of low molecular weight previously reported. ${ }^{27}$

In the low MS C+D fraction (Fig. 4) the trend is similar to that observed in the $\mathrm{B}$ fraction, i.e., a higher amount of oxygenated groups revealed by the difference band at 1517

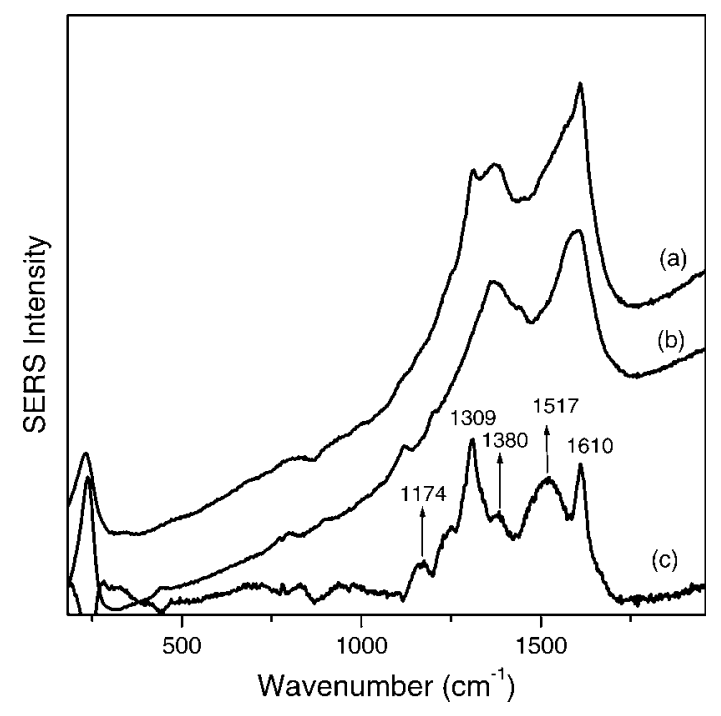

FIG. 3. SERS spectra of the B fraction at (a) alkaline and $(\boldsymbol{b})$ neutral pH; $(\boldsymbol{c})$ the difference between (a) and (b). 


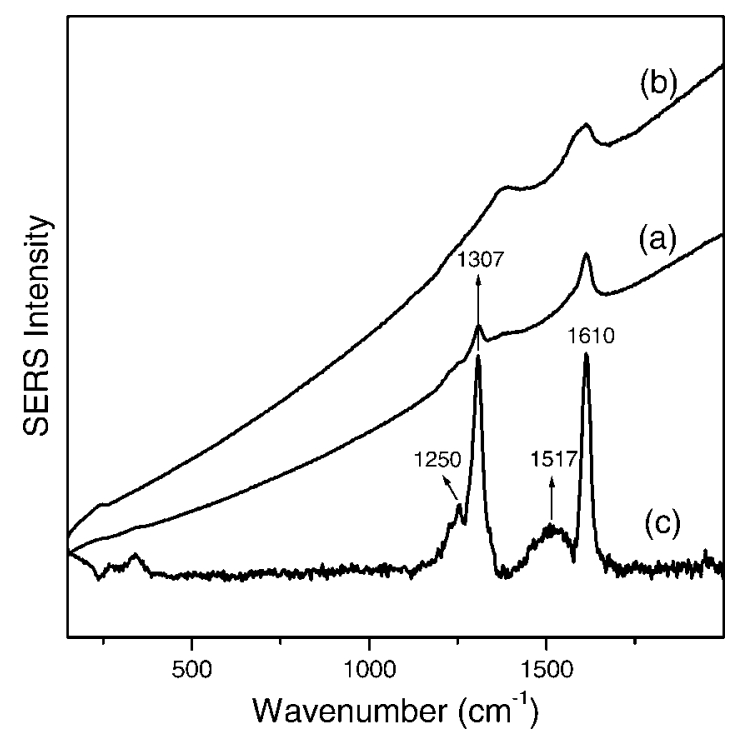

FIG. 4. SERS spectra of the $\mathrm{C}+\mathrm{D}$ fraction at $(\boldsymbol{a})$ alkaline and $(\boldsymbol{b})$ neutral $\mathrm{pH}$; (c) the difference between (a) and (b).

$\mathrm{cm}^{-1}$. An intense band at $1308 \mathrm{~cm}^{-1}$ is also observed, but it has an intensity similar to that at $1610 \mathrm{~cm}^{-1}$.

Finally, the SERS spectra of the bulk chernozem HA exhibit a large fluorescence background as in the case of the $\mathrm{C}+\mathrm{D}$ fraction, at both alkaline (Fig. 5a) and neutral pH (Fig. 5b), and stronger bands at 1610 and $1308 \mathrm{~cm}^{-1}$ at alkaline $\mathrm{pH}$, which are more evident in the difference spectrum (Fig. 5c).

\section{DISCUSSION}

$\mathrm{Li}$ et al. ${ }^{28}$ reported that large variations in chemical and molecular properties among different HA fractions were attributable to very different extractabilities of two chemically and structurally different subunits of HA. One subunit is aromatic and polar in nature and is highly soluble in alkaline solution whereas the other is aliphatic, less polar, and less soluble in alkaline solution.

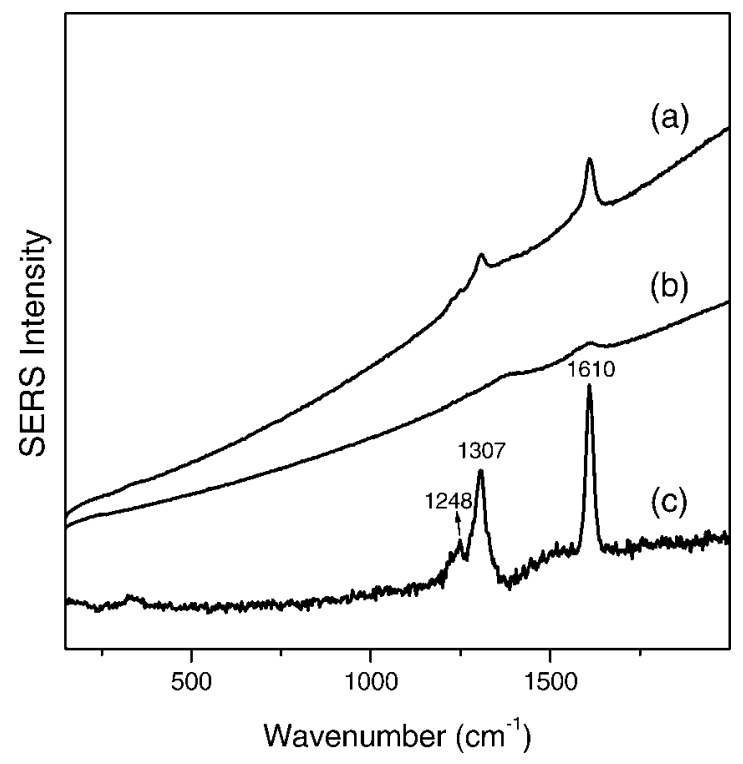

FIG. 5. SERS spectra of bulk HA at $(\boldsymbol{a})$ alkaline and $(\boldsymbol{b})$ neutral $\mathrm{pH} ;(\boldsymbol{c})$ the difference between (a) and (b).
The remarkable increase of bands at 1610 and $1308 \mathrm{~cm}^{-1}$ in going from neutral to alkaline $\mathrm{pH}$ in SERS spectra is attributed to a structural change occurring in the high MS fraction A. To understand this change it is assumed that at neutral $\mathrm{pH}$ a coil shrinking of the macromolecule occurs due to a protonation of the carboxylate groups, leading to an increase in the number of intermolecular $\mathrm{H}$-bonds inside the molecule. In addition, hydrophobic interactions established between less hydrophilic residues (aromatic and aliphatic ones), directed towards the inner side of the humic substance, could further destabilize the overall macromolecular structure. At alkaline $\mathrm{pH}$ an ionization of carboxylic groups is induced, leading to a massive breakdown of H-bonds and, subsequently, to the uncoiling of the humic acid structure. In fact the aromatic groups responsible for the bands at 1610 and $1308 \mathrm{~cm}^{-1}$ are then more exposed to the macromolecule outer part, as a consequence of the structural change occurring in the humic acid, as deduced from their intensity increase in the SERS spectra.

This hypothesis is supported by $\mathrm{H} / \mathrm{C}$ atomic ratio and thermochemolysis data (Tables I and II), which indicate that fraction $\mathrm{A}$ is more aliphatic than fractions $\mathrm{B}$ and $\mathrm{C}+\mathrm{D}$ and contains fewer polar (lignin) compounds. It was evident that fraction A contained considerably more fatty acids derived from plant waxes than fractions $\mathrm{B}$ and $\mathrm{C}+\mathrm{D}$. This higher amount of hydrophobic residues, which contribute to the further stabilization of the tri-dimensional structure of humic acids, could explain the stronger conformational changes seen for the A fraction deduced from the SERS spectra obtained at different $\mathrm{pH}$, in relation to the other fractions. On the other hand, it has been reported previously ${ }^{29}$ that at $\mathrm{pH}>9.5 \mathrm{HA}$ are flexible, linear polyelectrolytes and begin practical coil shrinking at $\mathrm{pH}<9.0$. On the basis of our data it should be suggested that probably due to the high MS and aliphatic character, fraction $\mathrm{A}$ is undergoing conformation changes versus fractions $\mathrm{B}$ and $\mathrm{C}+\mathrm{D}$, where this effect was not observed. It could suggest that fraction A is mostly responsible for the configuration changes during $\mathrm{pH}$ changes in the HA investigated.

The difference band observed in the SERS spectra of B and $\mathrm{C}+\mathrm{D}$ fractions at around $1517 \mathrm{~cm}^{-1}$ can be attributed to the $19 \mathrm{a}$ vibration of benzene groups ${ }^{30}$ and is related to the intense band usually appearing at around $1510 \mathrm{~cm}^{-1}$ in the infrared absorption spectra of aromatic compounds bearing a high amount of electron donor groups, such as hydroxyl and alkyl ethers. ${ }^{31}$ The Raman spectra of related aromatic compounds such as p-hydroxybenzoic acid ${ }^{32}$ and guaiacol ${ }^{33}$ also show a medium Raman band seen at $1517 \mathrm{~cm}^{-1}$. Moreover, intense Raman bands were found in the SERS spectra of caffeic acid, ${ }^{34}$ cathecol, ${ }^{35}$ gallic acid, ${ }^{36}$ and vanillic acid ${ }^{32}$ at 1505,1519 , 1515 , and $1537 \mathrm{~cm}^{-1}$, respectively. Some of these molecules were reported to have undergone a chemical change leading to lignin-like polyaromatic products, with a high content in oxygen groups, similar to those forming part of the low molecular weight humic substances. Thus, the intensity increase of the band centered at $1517 \mathrm{~cm}^{-1}$ can be considered a hint to the existence of lignin-like aromatic rings bearing a large amount of oxygenated groups in fractions $\mathrm{B}$ and $\mathrm{C}+\mathrm{D}$, as also revealed by thermochemolysis. This is corroborated by the fact that low molecular weight humic acid fractions, which are richer in oxygenated groups and contain a higher amount of lignin-like compounds, display a SERS band at $1516 \mathrm{~cm}^{-1} \cdot{ }^{37}$ 
As shown in this paper a correlation between thermochemolysis and SERS data was found, as the structural data explain the observed changes in the SERS spectra. The combination of a thermal destructive technique and a spectroscopic analysis is a good approach for characterizing structural components in HS.

\section{ACKNOWLEDGMENTS}

This work was supported by travel grants from the Consejo Superior de Investigaciones Cientificas-Russian Academy of Sciences Cooperation Agreement. Part of this research has been supported by INTAS (project 01-186) and Russian Foundation for Basic Research (project 04-05-64687-a), and part was supported by Dirección General de Investigación, Ministerio de Educación y Ciencia (grant FIS2004-00108), and Comunidad Autonoma de Madrid (project GR/MAT/0439/2004).

1. P. Schmitt-Kopplin, A. W. Garrison, E. M. Perdue, D. Freitag, and A. Kettrup, J. Chromatogr., A 807, 101 (1998).

2. M. De Nobili and Y. Chen, Soil Sci. 164, 825 (1999).

3. M. B. Muller, D. Schmitt, and F. H. Frimmel, Environ. Sci. Technol. 34, 4867 (2000).

4. P. Janoš, J. Chromatogr., A 983, 1 (2003).

5. T. Reemtsma and A. These, Anal. Chem. 75, 1500 (2003).

6. O. A. Trubetskoj and O. E. Trubetskaya, G. V. Afanas'eva, O. I. Reznikova, and C. Saiz-Jimenez, J. Chromatogr., A 767, 285 (1997).

7. O. E. Trubetskaya, G. V. Afanas'eva, O. I. Reznikova, L. F. Markova, T. A. Muranova, and O. A. Trubetskoj, Environ. Int. 24, 573 (1998).

8. C. Richard, O. A. Trubetskaya, O. E. Trubetskoj, O. I. Reznikova, G. V. Afanas'eva, J. P. Aguer, and G. Guyot, Environ. Sci. Technol. 38, 2052 (2004).

9. A. J. Simpson, J. Burdon, C. L. Graham, M. H. B. Hayes, N. Spencer, and W. L. Kingery, Eur. J. Soil Sci. 52, 495 (2001).

10. A. J. Simpson, W. L. Kingery, M. H. B. Hayes, M. Spraul, E. Humpfer, P. Dvortsak, R. Kerssebaum, M. Godejohann, and M. Hoffmann, Naturwissenschaften 89, 84 (2002).

11. C. Saiz-Jimenez, B. Hermosin, G. Guggenberger, and W. Zech, Eur. J. Soil. Sci. 47, 61 (1996).

12. P. Hildebrandt and M. Stockburger, J. Phys. Chem. 88, 5935 (1994).

13. M. Moskovits, Rev. Mod. Phys. 57, 783 (1985).

14. A. Wokaun, Mol. Phys. 56, 1 (1985).
15. O. Francioso, S. Sanchez-Cortes, V. Tugnoli, C. Ciavatta, L. Sitti, and C. Gessa, Appl. Spectrosc. 50, 1165 (1996).

16. O. Francioso, S. Sanchez-Cortes, V. Tugnoli, C. Ciavatta, and C. Gessa, Appl. Spectrosc. 55, 270 (1998).

17. E. Koglin and J. M. Sequaris, "Surface enhanced Raman scattering of biomolecules", in Topics in Current Chemistry, H. U. Borgstedt, Ed. (Springer Verlag, Berlin, 1986), pp. 1-57.

18. C. Saiz-Jimenez, O. E. Trubetskaya, O. A. Trubetskoj, and B. Hermosin, Comm. Soil Sci. Plant Anal. 30, 345 (1999).

19. O. A. Trubetskoj, L. Y. Kudryavceva, and L. T. Shirshova, Soil Biol. Biochem. 23, 1179 (1991).

20. C. Saiz-Jimenez, Environ. Sci. Technol. 28, 197 (1994).

21. C. Saiz-Jimenez, Environ. Sci. Technol. 28, 1773 (1994).

22. N. Leopold and B. Lendl, J. Phys. Chem. B 107, 5723 (2003).

23. S. Sanchez-Cortes, J. V. Garcia-Ramos, and G. Morcillo, J. Colloid Interface Sci. 167, 428 (1994).

24. S. Sanchez-Cortes, J. V. Garcia-Ramos, G. Morcillo, and A. Tinti, J. Colloid. Interface Sci. 175, 358 (1995).

25. C. Saiz-Jimenez, B. Hermosin, O. Trubetskaya, O. Reznikova, G. Afanas'eva, and O. Trubetskoj, Geoderma, paper in press (2005).

26. C. Saiz-Jimenez and J. W. de Leeuw, Org. Geochem. 6, 417 (1984).

27. S. Sanchez-Cortes, O. Francioso, C. Ciavatta, J. V. Garcia-Ramos, and C. Gessa, J. Colloid Interface Sci. 198, 308 (1998).

28. L. Li, W. Huang, P. Peng, G. Sheng, and J. Fu, Soil Sci. Soc. Am. J. 67, 740 (2003).

29. M. Schnitzer, Soil Sci. 451, 41 (1991).

30. G. Varsanyi, Assignments for Vibrational Spectra of Seven Hundred Benzene Derivatives (Akademiai Kiado and Adam Hilger LTD, London, 1974).

31. G. Socrates, Infrared and Raman Characteristic Group Frequencies: Tables and Charts (John Wiley and Sons, Chichester, 2001), 3rd ed.

32. S. Sanchez-Cortes and J. V. Garcia-Ramos, J. Colloid Interface Sci. 231, 98 (2001).

33. Bruker Analytische Messtechnik GMBH Library (1995).

34. S. Sanchez-Cortes and J. V. Garcia-Ramos, Appl. Spectrosc. 54, 230 (2000).

35. S. Sanchez-Cortes, O. Francioso, J. V. Garcia-Ramos, C. Ciavatta, and C. Gessa, Colloid Surf. A: Physicochem. Eng. Asp. 176, 77 (2001).

36. M. Alvarez-Ros, S. Sanchez-Cortes, O. Francioso, and J. V. GarciaRamos, J. Raman Spectrosc. 32, 143 (2001).

37. O. Francioso, S. Sanchez-Cortes, D. Casarini, J. V. Garcia-Ramos, C. Ciavatta, and C. Gessa, J. Mol. Struct. 609, 137 (2002). 